\title{
Comparison of changes in oxygenated hemoglobin during the tree-drawing task between patients with schizophrenia and healthy controls
}

This article was published in the following Dove Press journal:

Neuropsychiatric Disease and Treatment

\author{
Shinya Nakano ${ }^{1,2}$ \\ Yoshihisa Shoji 1,3 \\ Kiichiro Morita ${ }^{1,3}$ \\ Hiroyasu Igimi ${ }^{1,4}$ \\ Mamoru Sato ${ }^{1,3}$ \\ Youhei Ishii' \\ Akihiko Kondo' \\ Naohisa Uchimura ${ }^{1,3}$ \\ 'Cognitive and Molecular Research \\ Institute of Brain Diseases, \\ Kurume University, Kurume, Japan; \\ ${ }^{2}$ Department of Clinical Laboratory \\ Medicine, Kurume University \\ Hospital, Kurume, Japan; ${ }^{3}$ Department \\ of Neuropsychiatry, Kurume \\ University School of Medicine, \\ Kurume, Japan; ${ }^{4}$ Department of \\ Neuropsychiatry, Horikawa Hospital, \\ Medical Corporation Association \\ Horikawakai, Kurume, Japan
}

Correspondence: Yoshihisa Shoji Department of Neuropsychiatry, Kurume University School of Medicine, 67, Asahi-Machi, Kurume 830 00II, Japan

Tel +8 I 9423 3 758 I

Fax +81942317682

Email yshoji@med.kurume-u.ac.jp
Background: Tree-drawing test is used as a projective psychological test that expresses the abnormal internal experience in patients with schizophrenia (SZ). Despite the widely accepted view that the cognitive function is involved in characteristic tree-drawing in patients with SZ, no study has psychophysiologically examined it. The present study aimed to investigate the involvement of cognitive function during tree-drawing in patients with SZ. For that purpose, we evaluated the brain function in patients with SZ during a tree-drawing task by using nearinfrared spectroscopy (NIRS) and compared them with those in healthy controls.

Patients and methods: The subjects were 28 healthy controls and 28 patients with SZ. Changes in the oxygenated hemoglobin $([\mathrm{oxy}-\mathrm{Hb}])$ concentration in both the groups during the task of drawing a tree imagined freely (free-drawing task) and the task of copying an illustration of a tree (copying task) were measured by using NIRS.

Results: Because of the difference between the task conditions, [oxy-Hb] levels in controls during the free-drawing task were higher than that during the copying task at the bilateral frontal pole regions and left inferior frontal region. Because of the difference between the groups, [oxy-Hb] levels at the left middle frontal region, bilateral inferior frontal regions, bilateral inferior parietal regions, and left superior temporal region during the free-drawing task in patients were lower than that in controls.

Conclusion: [oxy-Hb] during the tree-drawing task in patients with $\mathrm{SZ}$ was lower than that in healthy controls. Our results suggest that brain dysfunction in patients with SZ might be associated with their tree-drawing.

Keywords: tree-drawing test, near-infrared spectroscopy, cognitive function, mnemonic retrieval, executive function, working memory, attention

\section{Introduction}

Schizophrenia (SZ) is a psychiatric disorder showing positive symptoms, such as hallucination and delusion, and negative symptoms such as a disorder of affect and motivation. Recently, the importance of cognitive impairment has also been indicated. ${ }^{1,2}$

The tree-drawing (Baum) test is a useful, systematized, projective psychological test that involves instructing patients to draw a fruit-bearing tree carefully based on the original method of Koch. ${ }^{3}$ It is considered that the test can detect an abnormal internal experience with a reduced burden on patients. This test is often used in patients with $\mathrm{SZ}$, and it has been reported that such patients draw pathognomonic trees, such as branches and trunk bases being open, compared with healthy subjects. ${ }^{4}$ However, since subjects perform this test based on their own free will and their drawings widely vary, objective analysis is difficult. At this point, the question arises of why tree-drawing 
in patients with SZ tends to be different from that in healthy subjects. However, it has been mainly studied from the viewpoints of psychology and psychopathology, because it is considered as a projective psychological test. On the other hand, Stanzani et al examined the cognitive function and drawing contents of the tree-drawing test in patients with dementia and reported that the tree height and trunk-to-crown ratio are predictors of cognitive impairment. ${ }^{5}$ Therefore, we considered that the cognitive function might be involved in the characteristic tree-drawing in patients with $\mathrm{SZ}$ and thought that it was necessary to investigate this possibility psychophysiologically.

Regarding the relationship between drawing and cognitive function, a study involving infants reported that the executive function and capacity of working memory (WM) play important roles in the early development of drawing flexibility. ${ }^{6}$ Since the tree-drawing test involves drawing by imagining a completed tree, a subject should perform the task while appropriately controlling behaviors to achieve the goal. Therefore, we must also consider the involvement of WM and attention/execution functions in tree-drawing. Furthermore, a drawing of a tree is elicited from individual mnemonic representations or past context, being a form of mnemonic retrieval. Regarding mnemonic retrieval, Tulving et al reviewed previous studies and reported that the frontal lobe advances the coding and retrieval of episodic memory. ${ }^{7}$ In addition, functional magnetic resonance imaging (fMRI) studies that examined the brain function during mentally generating specific images reported activation in the parietal and superior temporal cortex. ${ }^{8-11}$ However, due to restrictions on fMRI measurement, the tasks in those studies do not represent typical drawing conditions.

Near-infrared spectroscopy (NIRS) can be used for the noninvasive determination of relative concentrations of oxygenated hemoglobin ([oxy-Hb]) and deoxygenated hemoglobin ([deoxy-Hb]) in blood vessels on the surface of the brain by using scattering light of near-infrared rays. Although its spatial resolution is lower $(2-3 \mathrm{~cm})$, its temporal resolution is more favorable ( 0.1 seconds) than that of other blood flow evaluation methods. Furthermore, NIRS involves a relatively small and portable apparatus, and it is possible to easily measure neural activation of the cerebral cortex; thus, many studies have recently been performed by using this method. ${ }^{12-14}$ NIRS has also been applied to studies of the cognitive function in patients with SZ, and it was revealed that activation of the prefrontal cortex associated with attention/executive function and WM decrease during the trail-making test (TMT) and verbal fluency task. ${ }^{15,16}$ In addition, subjects are not restrained in a gantry, such as in
fMRI, and so NIRS can be applied to a natural experimental setting, and it is suitable for measuring the hemodynamic response during drawing such as tree-drawing. Shoyama et al used NIRS and observed frontal and temporal activation during clock-drawing. ${ }^{17}$ Furthermore, an NIRS study involving healthy subjects by Igimi compared the brain activation on drawing an imagined tree with that on copying an illustration of a tree. ${ }^{18}$ Based on the results, he suggested that WM is important in understanding the physiological mechanism of tree-drawing, since the activities of the left frontal region and right parietal region increase more in the former task. However, no study has psychophysiologically compared the results of the tree-drawing test in patients with SZ and healthy controls.

Therefore, in this study, we investigated the possibility that the cognitive function is involved in characteristic tree-drawing by patients with SZ. For that purpose, we psychophysiologically evaluated the brain function of patients with SZ by using the tasks of drawing a tree imagined freely and copying an illustration of a tree combined with NIRS and then compared the results with those of healthy controls. We hypothesized that brain regions activated in healthy controls during the tree-drawing task would not be activated in patients with SZ.

\section{Patients and methods Subjects}

We examined 28 patients with SZ (14 male and 14 female patients, age: $30.8 \pm 5.3$ years) and 28 healthy age- and sexmatched volunteers (14 male and 14 female participants, age: $30.8 \pm 5.1$ years) by multi-channel NIRS during the treedrawing task. All the patients were diagnosed using the Diagnostic and Statistical Manual of Mental Disorders, fourth Edition, Text Revision (DSM-IV-TR). ${ }^{19}$ They had been treated as outpatients at the Department of Psychiatry, Kurume University Hospital. Their intelligence quotient (IQ) was estimated using the Japanese version of the National Adult Reading Test. ${ }^{20}$ All the patients were taking atypical antipsychotic medication (ie, olanzapine [ $\mathrm{n}=8]$, risperidone $[\mathrm{n}=14]$, aripiprazole [ $\mathrm{n}=2]$, quetiapine [ $\mathrm{n}=2]$, and blonanserin [ $\mathrm{n}=6]$ ). The mean daily antipsychotic dose expressed as a haloperidol equivalent was $8.1 \pm 2.4 \mathrm{mg}$. The clinical state of the patients was assessed by using the Positive and Negative Symptom Scale (PANSS) by two psychiatrists within 1 week after NIRS recording. ${ }^{21}$ No controls had a history of psychiatric or neurological diseases, cerebrovascular disease, brain injury, or substance abuse including alcohol. All the subjects were right-handed as determined by the Edinburgh Handedness Inventory $^{22}$ and had normal vision. The Ethics Committee 
Table I Demographic and clinical characteristics of controls and patients

\begin{tabular}{llll}
\hline & Controls & Patients & P-value \\
\hline $\mathrm{N}$ & 28 & 28 & \\
Age (years) & $30.8 \pm 5.1$ & $30.8 \pm 5.3$ & NS \\
Sex (female/male) & $14 / 14$ & $14 / 14$ & \\
Estimated IQ (JART) & $103.4 \pm 8.9$ & $97.2 \pm 12.8$ & 0.04 \\
Educational history (years) & $14.9 \pm 0.67$ & $14.25 \pm 2.4$ & NS \\
Duration of illness (years) & & $6.6 \pm 4.4$ & \\
PANSS & & & \\
$\quad$ Positive score & & $23.4 \pm 3.7$ & \\
$\quad$ Negative score & & $21.1 \pm 4.1$ & \\
Haloperidol equivalent & & $8.3 \pm 2.2$ & \\
dose (mg/day) & & & \\
\hline
\end{tabular}

Notes: Data are presented as means $\pm S D$. An unpaired $t$-test was used for testing group differences.

Abbreviations: IQ, intelligence quotient; JART, the Japanese version of the National Adult Reading Test; PANSS, Positive and Negative Syndrome Scale; NS, not significant.

of Kurume University approved this study (No 10086), and written informed consent was obtained from all the subjects prior to the study. The demographic and clinical characteristics of the subjects are shown in Table 1.

\section{Protocol of the free-drawing and tree- copying tasks}

In the present study, we used two types of tree-drawing tasks following the method of Igimi. ${ }^{18}$ One was the task of drawing a tree imagined freely (free-drawing task) and the other was the task of copying an illustration of a tree (copying task). The copying task was used to examine and compare the element of imagination or mnemonic retrieval with that of the free-drawing task. In the free-drawing task, all the subjects were instructed to "Draw a fruit-bearing tree carefully," and they drew a tree from their imagination in an A4 paper with a 4B pencil. In the copying task, the subjects began to copy a tree illustration in front of them after instruction. Based on Igimi and Fujiki et al, ${ }^{18,23}$ the task period in the present study was set at 60 seconds (Figure 1). For the control conditions of both tasks as the baseline, all the subjects continued to draw " $O$ " and " $X$ " for 60 seconds. The task and control conditions were alternately repeated four times. Drawing was started from the beginning every time. Typical examples of a tree illustration as a model in the copying task and drawings in each task are shown in Figure 2.

A
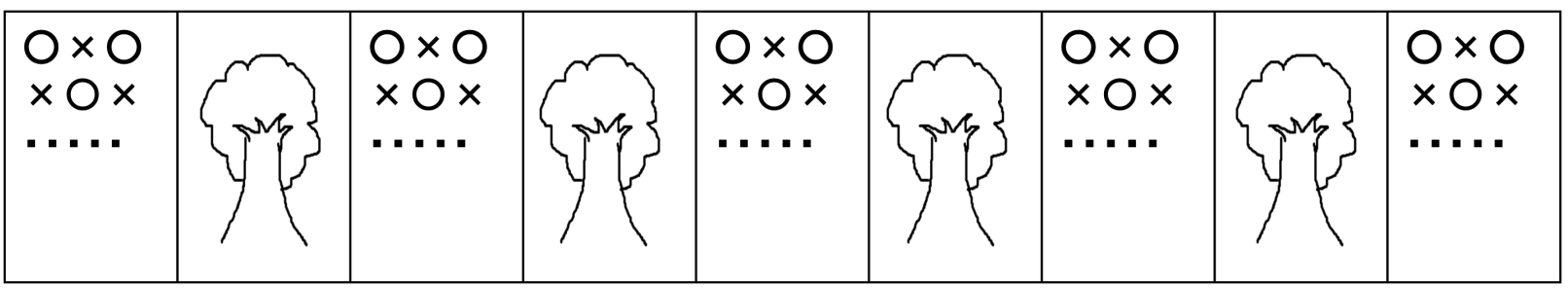

B

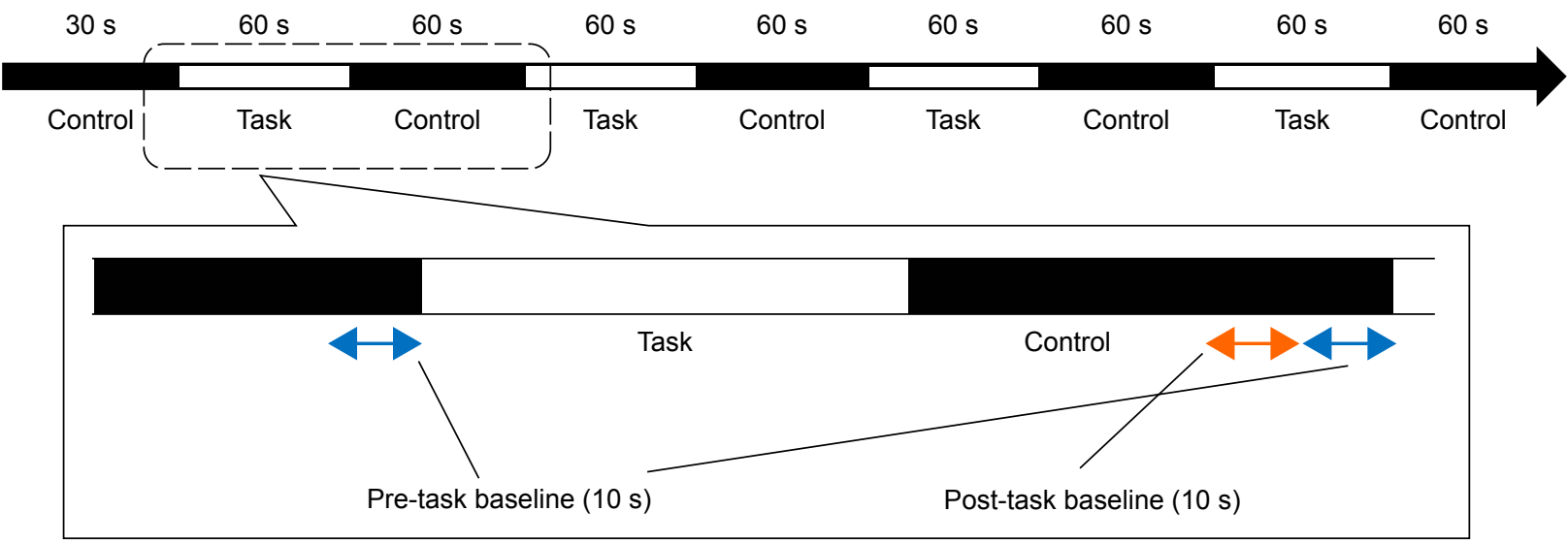

C $\left[\mathrm{mM}^{*} \mathrm{~mm}\right]$

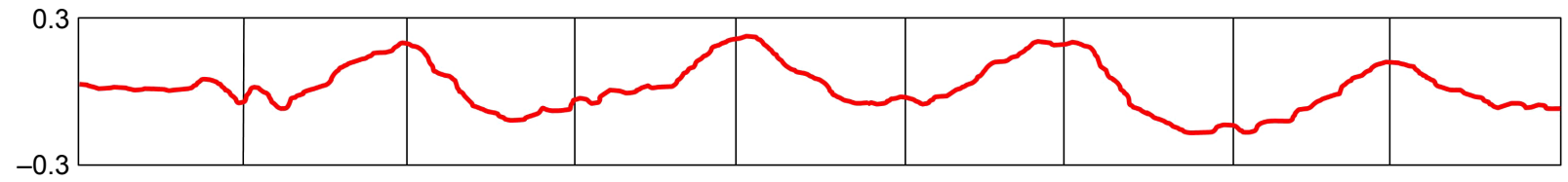

Figure I Task design.

Notes: (A) The task composition. During rest periods as control conditions, subjects continued to draw " $\bigcirc$ " and " $\times$ " for 60 seconds. During task periods, subjects drew an imagined tree or copied an illustration of a tree for 60 seconds. Each task was repeated four times. (B) The detail of periods in the baseline processing. Blue arrows show the pre-task baseline. Orange arrows show the post-task baseline. (C) A typical waveform of [oxy- $\mathrm{Hb}$ ] in a healthy subject during the free-drawing task. 


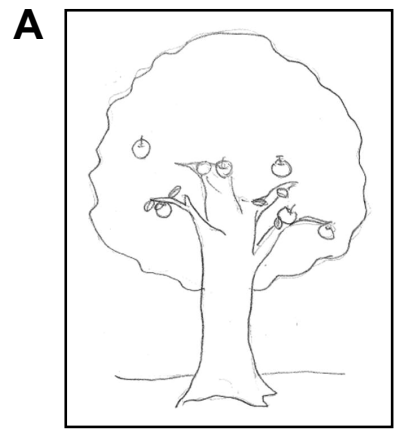

B

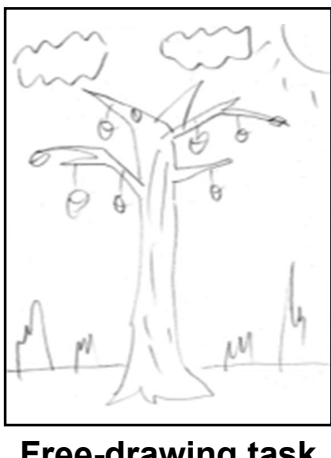

Free-drawing task

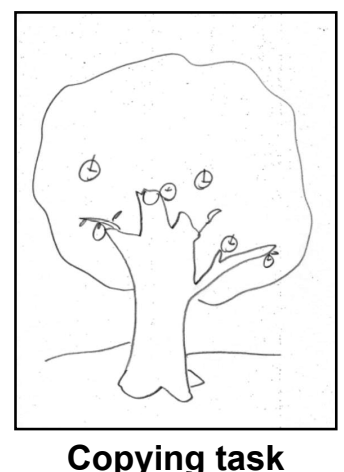

Copying task

A healthy subject
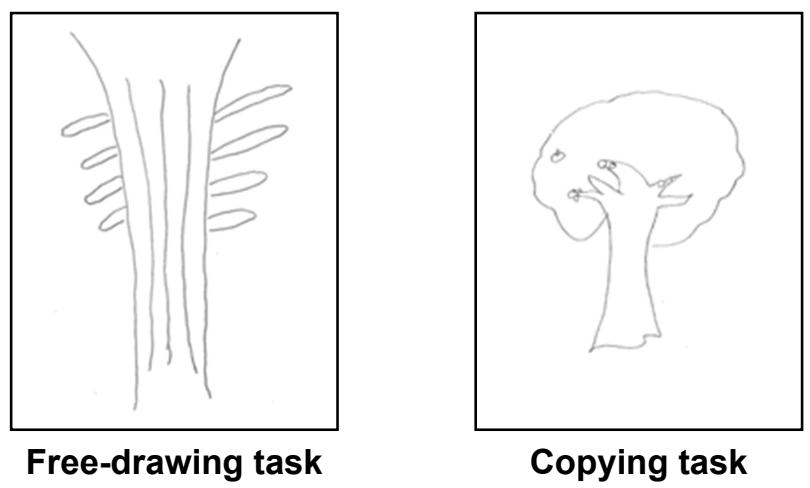

Copying task

A patient

Figure 2 Examples of tree-drawings.

Notes: (A) A typical example of a tree illustration as a model in the copying task. (B) Typical examples of drawings made by a healthy subject and a patient in the freedrawing and copying tasks.

\section{NIRS measurement}

The probe was placed so that the row of the most anteroinferior channels was present on the line connecting T2-FPZ-T4 in the international 10-20 system. NIRS measurements were conducted with the ETG-4000 Optical Topography System (Hitachi Medical Co., Kashiwa, Japan) using a total of 44 channel arrays of optodes that covered an area of $12 \times 6 \mathrm{~cm}$ on one side of the head. The distance between optodes was $30 \mathrm{~mm}$. Near-infrared light with wavelengths of $695-830 \mathrm{~nm}$ was used to irradiate the subject's scalp. The reflected light was detected by highly sensitive photodetectors that were also attached to the scalp surface. During the NIRS measurement, the jaw of subjects was fixed using a chin stand to prevent changes in blood flow due to head movement (Figure 3A). With reference to previous studies, ${ }^{18,23}$ the probe position was decided based on the association between the channel showing changes in [oxy-Hb] level during a right-hand motion task drawing "OX" and the anatomical region. In the present study, consistent with the result of Igimi using the same method, ${ }^{18}$ left channel (Ch) 3 was considered to correspond to the primary motor cortex (Brodmann area $[\mathrm{BA}] 4$ ). The baseline processing of the obtained data was executed based on a previous study..$^{15}$ Using the integral mode, the pre-task baseline was set as the mean during the 10 seconds preceding the task period, and the post-task baseline was set as the mean during the 10 seconds from 40 to 50 seconds after the task period (Figure 1). Linear fitting was then applied to the data between these two baselines. For the analysis of the task, average waveforms of the changes in [oxy-Hb] during tasks for 60 seconds that were performed four times were prepared and the approximate area $(\delta[\mathrm{oxy}-\mathrm{Hb}])$ based on changes in [oxy-Hb] for each $100 \mathrm{~ms}$ was used (Figure 3B). Regarding the relationship between each channel and anatomical regions, NIRS data were converted to a normalized brain image template (three-dimensional composition indication unit; Hitachi Medical Co.). We determined the region of interest (ROI) to be analyzed as the frontal pole regions (left Ch19 and right Ch22), middle frontal regions (left Ch11 and right Ch12), and inferior parietal regions (left $\mathrm{Ch} 9$ and right $\mathrm{Ch} 5$ ) related to the visual WM and executive function considered to be necessary for tree-drawing. Furthermore, we considered the inferior frontal regions (left $\mathrm{Ch} 20$ and 
A

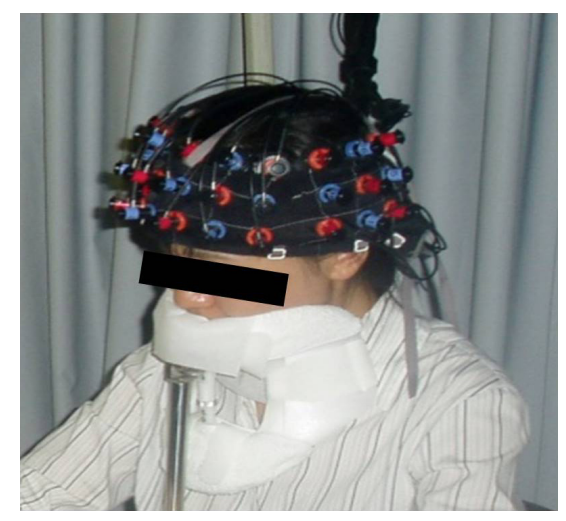

B

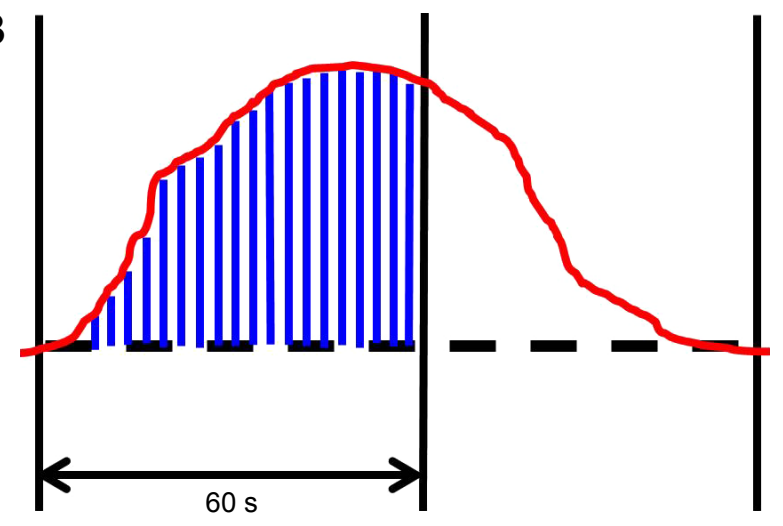

C

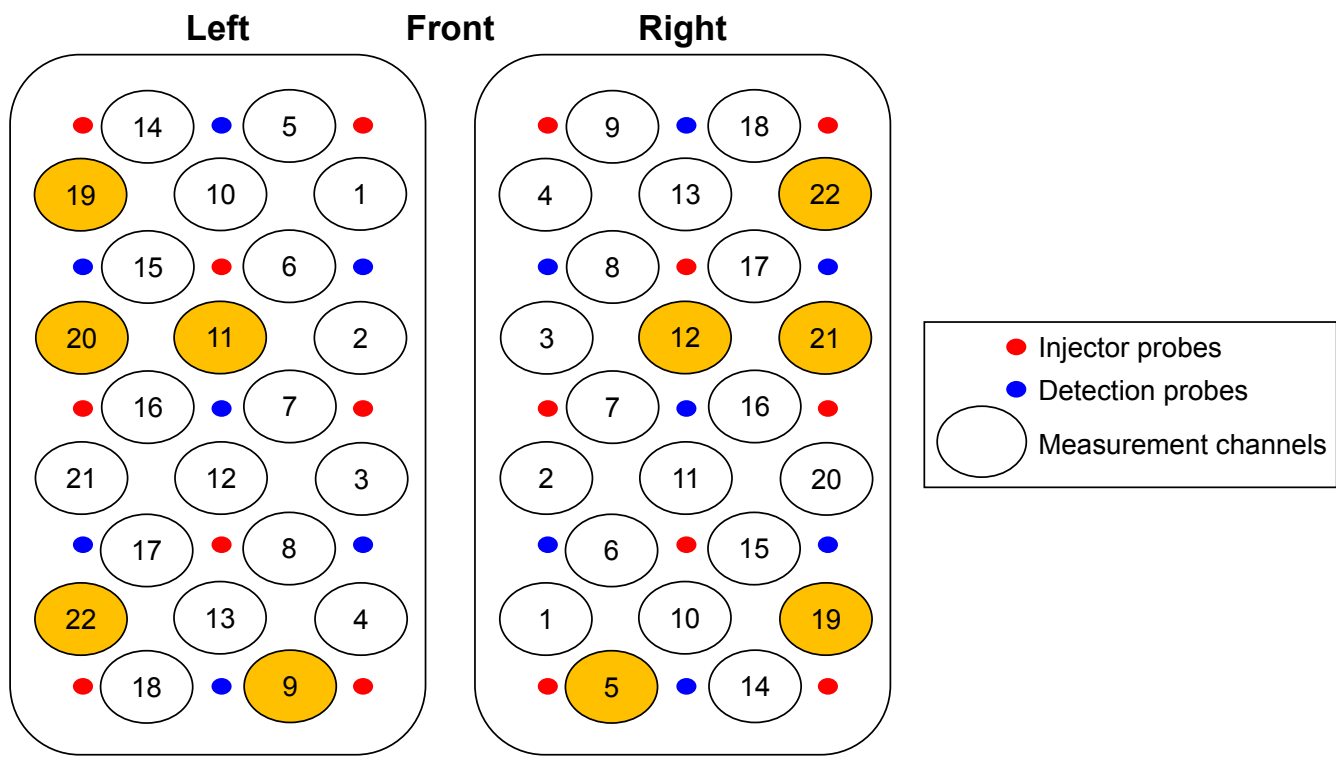

Back

Figure 3 NIRS measurement and analysis.

Notes: (A) The probe holder worn by a patient. The jaw was fixed using a chin stand to prevent changes in blood flow due to the head movement. (B) Data were calculated from the mean wave of changes in [oxy-Hb]. We calculated the area of the waveform of changes in [oxy- $\mathrm{Hb}$ ] during 60 seconds as the task period and used values of this area as NIRS data. (C) Location of channels and ROls. ROls are left Ch 19 and right Ch22; frontal pole regions, left ChI I and right ChI2; middle frontal regions, left Ch20 and right $\mathrm{Ch} 2 \mathrm{I}$; inferior frontal regions, left $\mathrm{Ch} 9$ and right Ch5; inferior parietal regions, left Ch22 and right Chl9; superior temporal regions. ROls are shown by yellow channels.

Abbreviations: ROI, region of interest; NIRS, near-infrared spectroscopy; [oxy-Hb], oxygenated hemoglobin.

right Ch21) and superior temporal regions (left Ch22 and right $\mathrm{Ch} 19)$ as the regions associated with retrieval of memory or visual meaning (Figure 3C).

\section{Statistical analysis}

In the present study, differences in demographic or clinical data between patients and controls were examined using Bartlet's $t$-test (Table 1). Two-way analysis of variance (ANOVA) for $\delta[0 x y-H b]$ in each ROI was performed, 2 (group: controls, patients) $\times 2$ (task condition: free-drawing task, copying task). If ANOVA identified a main effect, a post hoc test was conducted to examine it in more detail. At that time, in order to minimize the probability of being incorrectly significant (family-wise type I error rate), the
$P$-value was corrected by the Bonferroni method. Furthermore, the correlation between $\delta[0 x y-H b]$ and the symptom score of PANSS was calculated using Pearson's correlation coefficient. A significance level of $P<0.05$ was used for the analysis. All statistical processing was done using SPSS for Windows, version 22.0 (IBM Corporation, Armonk, NY, USA).

\section{Results \\ Cortical activation during tasks in each ROI}

The average waveforms in the controls and patients during each task are shown in Figure 4A and B. 
A

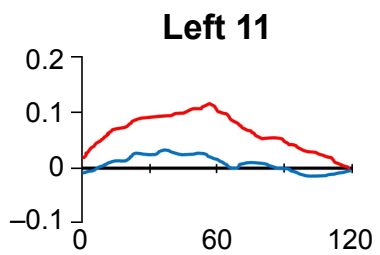

Left 20

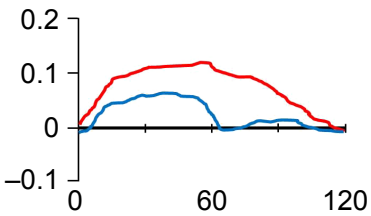

Left 9

$[\mathrm{mM} * \mathrm{~mm} * 60 \mathrm{~s}]$

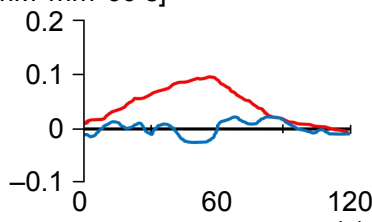

Right 5

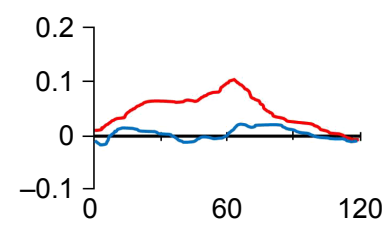

(s)
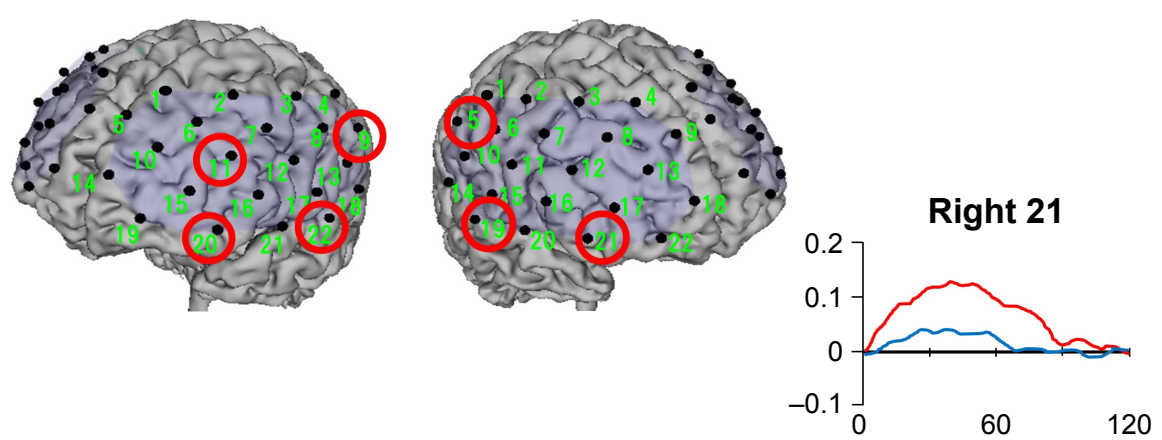

Left 22

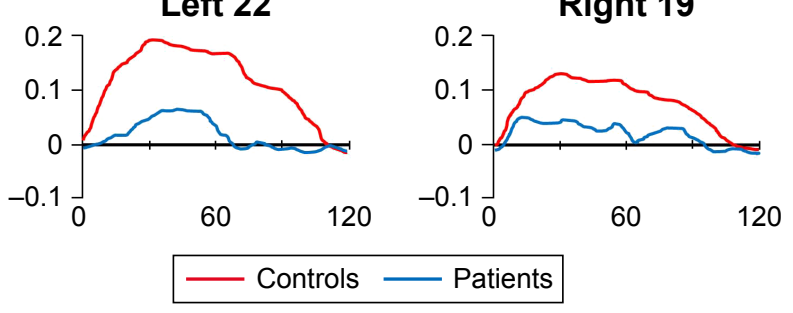

\section{B}

\section{Left 9}

$[\mathrm{mM} * \mathrm{~mm} * 60 \mathrm{~s}]$

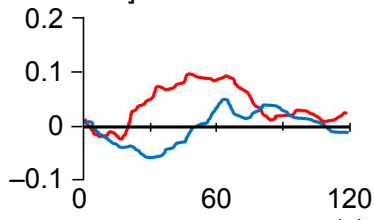

(s)

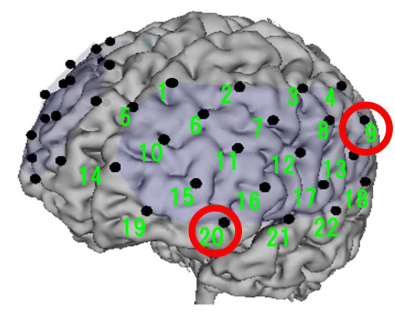

Left 20

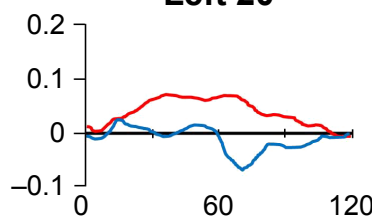

Right 21
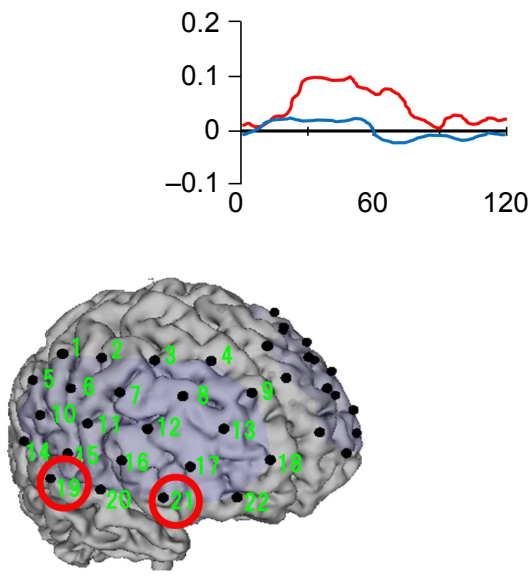

Right 19

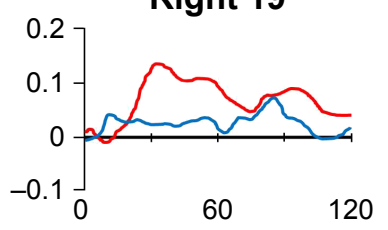

Figure 4 Average waveforms in the region of interest where significant differences were observed between controls and patients.

Notes: (A) Average waveforms during the free-drawing task in each group. (B) Average waveforms during the copying task in each group. The red lines represent controls, and the blue lines represent patients. Green numbers surrounded by the red circles indicate channels where the significant difference is observed, and they correspond to each figure of waveform. The longitudinal axis indicates the task period from 0 to 60 seconds, and the rest period from 60 to 120 seconds. 


\section{Consideration of $\delta[0 x y-\mathrm{Hb}]$ in each ROI}

The results of ANOVA in each ROI are shown below.

No interaction was observed in any ROIs (Figure 5).

\section{The frontal pole region}

Significant main effects of the task condition were shown at the left and right frontal pole regions (left Ch19, right Ch22) $(\mathrm{F}=13.563, P<0.001 ; \mathrm{F}=6.787, P=0.010)$. Based on the post hoc test, in the control group, $\delta[\mathrm{oxy}-\mathrm{Hb}]$ at the bilateral frontal pole regions during the free-drawing task was higher than that during the copying task $(\mathrm{F}=12.651$, $P=0.001 ; \mathrm{F}=8.288, P=0.005$ ). However, there was no significant difference between conditions in $\delta[\mathrm{oxy}-\mathrm{Hb}]$ at the left and right frontal pole regions of patients $(\mathrm{F}=2.728, P=0.102$; $\mathrm{F}=0.649, P=0.422$ ).

\section{The middle frontal region}

A significant main effect of group was shown only in the left middle frontal region (left Ch11) $(\mathrm{F}=8.605, P=0.004)$. The post hoc test revealed that $\delta[\mathrm{oxy}-\mathrm{Hb}]$ in patients during the free-drawing task was lower than that in controls $(\mathrm{F}=7.918$, $P=0.006$ ).

\section{The inferior frontal region}

In the left inferior frontal region (left Ch20), significant main effects of the task condition and group were observed (F=20.199, $P<0.001 ; \mathrm{F}=4.298, P=0.041$ ). As a result of the post hoc test, in controls, $\delta[\mathrm{oxy}-\mathrm{Hb}]$ during the free-drawing task was higher than that during the copying task $(\mathrm{F}=5.331$, $P=0.023)$. Also, during both the free-drawing and copying tasks, $\delta[\mathrm{oxy}-\mathrm{Hb}]$ in patients was lower than that in controls (F=16.167, $P<0.001 ; \mathrm{F}=5.453, P=0.021$ ). Only the main effect of group was shown in the right inferior frontal region (right $\mathrm{Ch} 21)(\mathrm{F}=15.513, P<0.001)$. As a result of the post hoc test, during both the tasks, $\delta[\mathrm{oxy}-\mathrm{Hb}]$ in patients was lower than that in controls $(\mathrm{F}=8.030, P=0.005 ; \mathrm{F}=7.491, P=0.007)$.

\section{The inferior parietal region}

Only the main effects of group were significant at the bilateral inferior parietal regions (left $\mathrm{Ch} 9$, right $\mathrm{Ch} 5)(\mathrm{F}=16.076$,

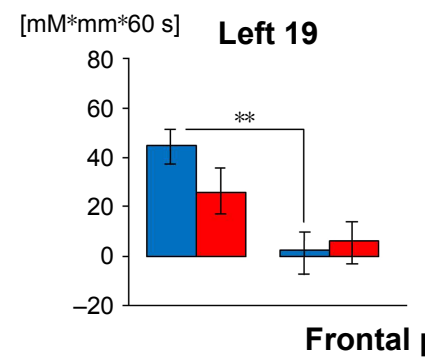

Left 20

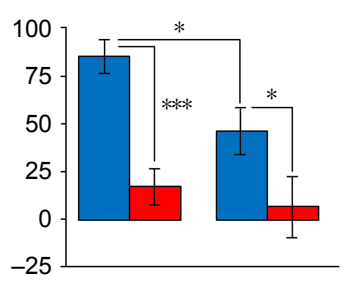

Right 22

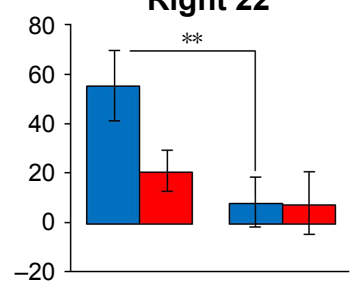

pole regions

Inferior frontal regions

Right 21

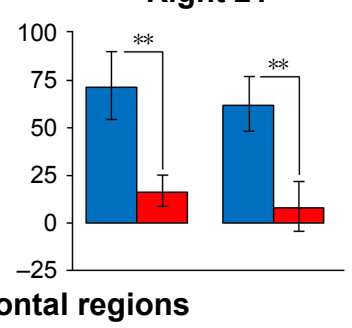

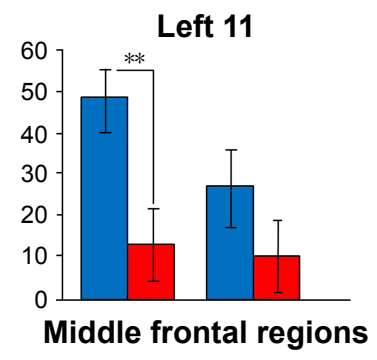

Left 9

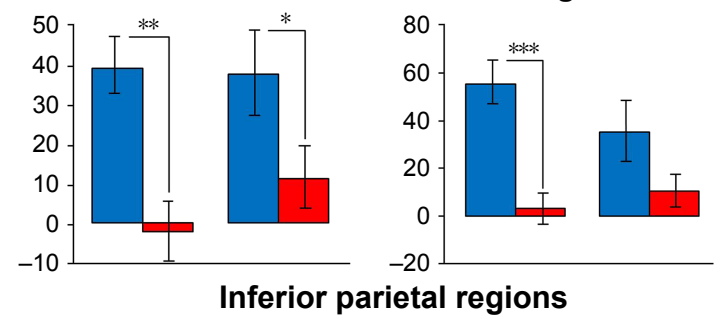

Left 22

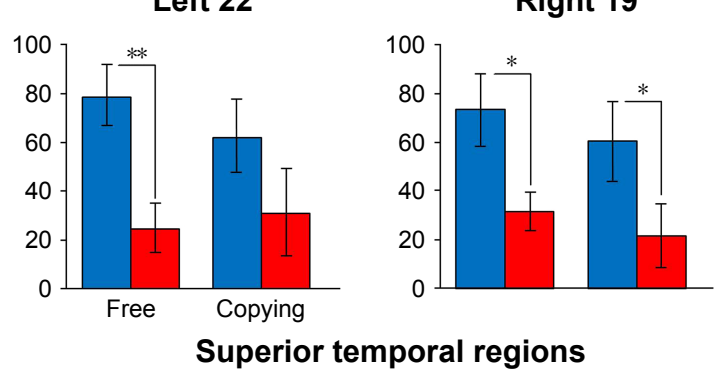

Right 19

Figure $\mathbf{5} \delta[\mathrm{oxy}-\mathrm{Hb}]$ in regions showing significant differences in groups or conditions.

Notes: The blue bars represents controls, and the red bars represent patients. Left two bars show $\delta$ [oxy- $\mathrm{Hb}$ ] during the free-drawing task; right two bars show $\delta[0 x y-\mathrm{Hb}]$ during the copying task. Error bars indicate \pm I standard error. Significance $* P<0.05, * * P<0.01, * * * P<0.001$.

Abbreviation: [oxy-Hb], oxygenated hemoglobin. 
$P<0.001 ; \mathrm{F}=18.116, P<0.001)$. As a result of the post hoc test, during both the free-drawing and copying tasks, $\delta[\mathrm{oxy}-\mathrm{Hb}]$ at the left inferior parietal region in patients was lower than that in controls $(\mathrm{F}=11.885, P=0.001 ; \mathrm{F}=4.916$, $P=0.029)$. A lower $\delta[\mathrm{oxy}-\mathrm{Hb}]$ at the right inferior parietal region in patients compared with controls was only noted in the free-drawing task $(\mathrm{F}=16.715, P<0.001)$.

\section{The superior temporal region}

Only the main effects of group were significant at the bilateral superior temporal regions (left Ch22, right Ch19) ( $\mathrm{F}=9.245, P=0.003 ; \mathrm{F}=8.742, P=0.004)$. As a result of the post hoc test, only $\delta[\mathrm{oxy}-\mathrm{Hb}]$ at the left superior temporal region in patients during the free-drawing task was lower than that in controls $(\mathrm{F}=7.455, P=0.007)$. Also, $\delta[\mathrm{oxy}-\mathrm{Hb}]$ at the right superior temporal region in patients during both

the tasks was lower than that in controls $(\mathrm{F}=4.667, P=0.033$; $\mathrm{F}=4.082, P=0.046)$.

\section{Relationship between symptoms and $\delta[\mathrm{oxy}-\mathrm{Hb}]$}

During the free-drawing task, the positive symptom score of PANSS was significantly and positively correlated with $\delta[\mathrm{oxy}-\mathrm{Hb}]$ at the left inferior frontal region (left $\mathrm{Ch} 20$ ) (r=0.410, $P=0.030$ ) (Figure 6). Furthermore, a significant positive correlation was noted between the negative symptom score and $\delta[\mathrm{oxy}-\mathrm{Hb}]$ at the left inferior frontal region (left $\mathrm{Ch} 20)$ ( $\mathrm{r}=0.406, P=0.032)$. During the copying task, the positive symptom score was significantly and negatively correlated with $\delta[\mathrm{oxy}-\mathrm{Hb}]$ at the left inferior parietal region (left $\mathrm{Ch} 9)(\mathrm{r}=-0.482, P=0.009)$. Also, the negative symptom score was significantly and negatively correlated

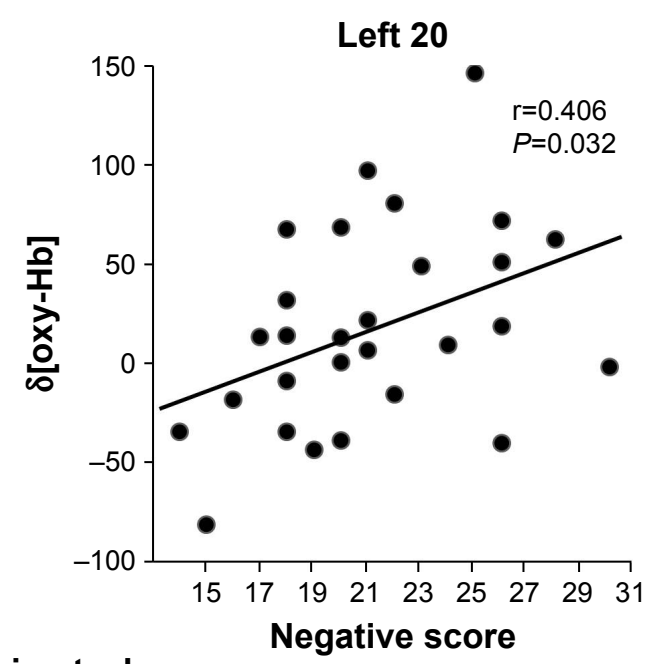

\section{Free-drawing task}
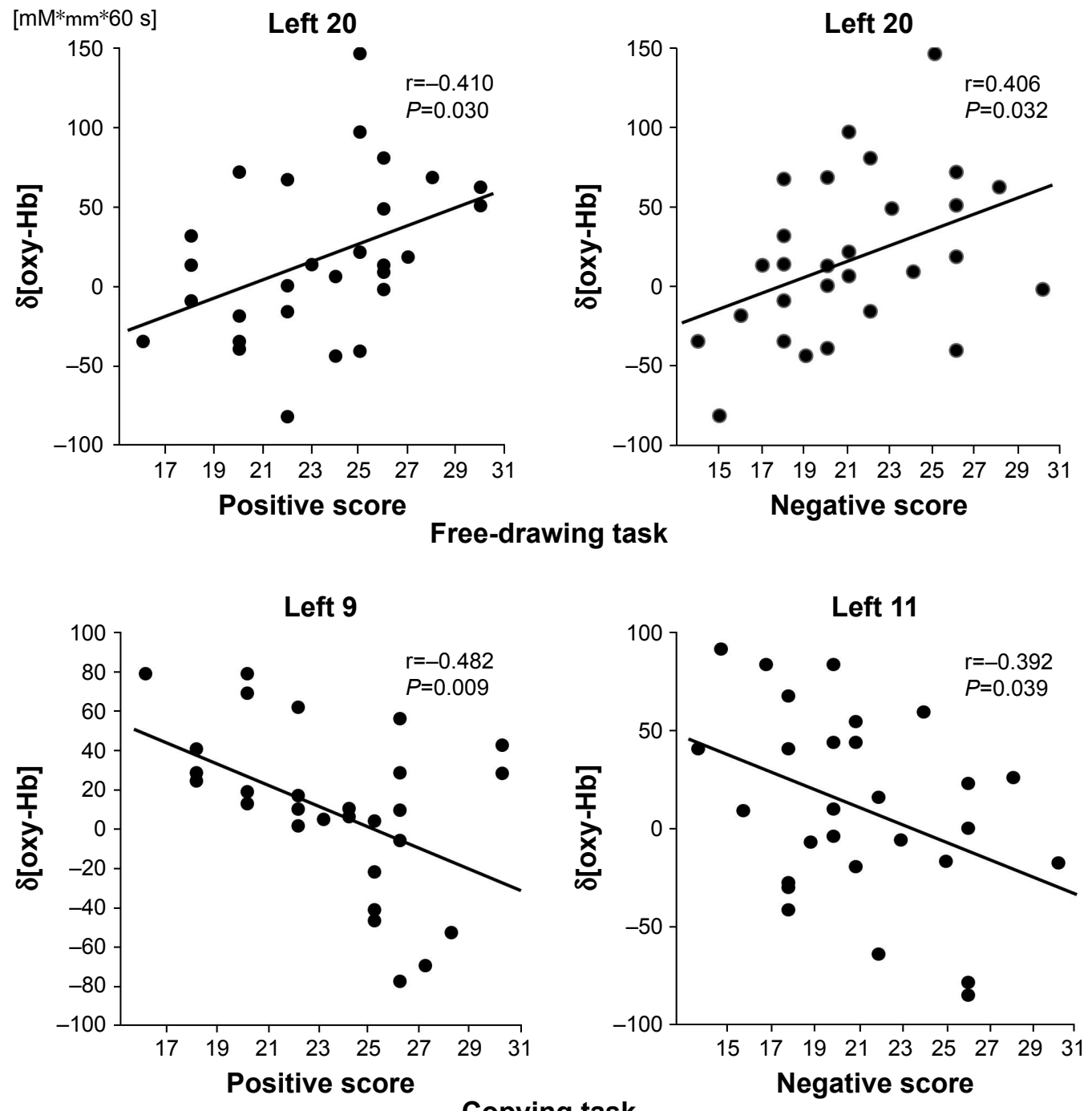

\section{Copying task}

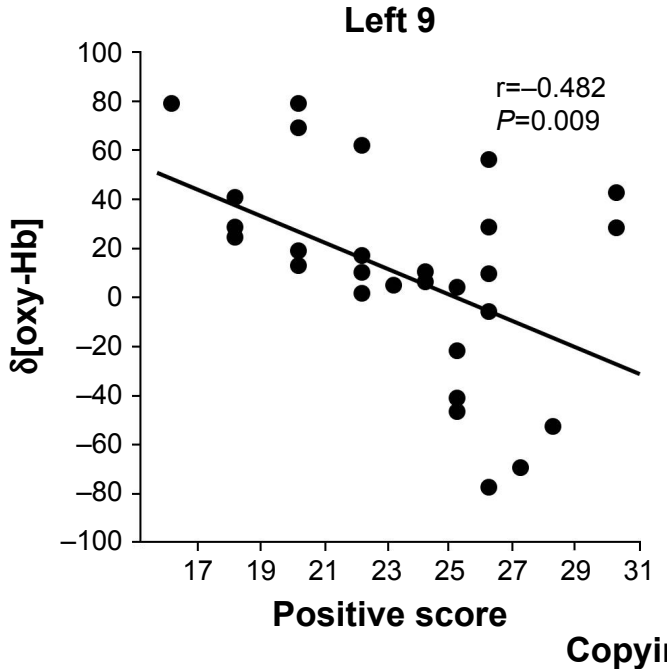

Figure 6 Scatter diagrams of regions showing significant correlations between $\delta[0 x y-H b]$ and Positive and Negative Symptom Scale scores. Abbreviation: [oxy-Hb], oxygenated hemoglobin. 
with $\delta[\mathrm{oxy}-\mathrm{Hb}]$ at the left middle frontal region (left Ch11) $(\mathrm{r}=-0.392, P=0.039)$.

\section{Discussion}

The main purpose of the present study was to investigate the involvement of the cognitive function in the tree-drawing test conducted by patients with SZ. We measured $\delta[0 x y-H b]$, reflecting the brain activation, in patients with SZ and healthy controls during free-drawing and copying tasks using NIRS. To our knowledge, this is the first study to identify a difference between patients with $\mathrm{SZ}$ and healthy controls regarding the hemodynamic response during tree-drawing. In the present study, the brain activation in patients was shown to be lower over a wide range compared with that of controls during both the free-drawing and copying tasks.

Regarding the main findings of the present study, we discuss as follows from the perspective of brain regions considered to be important. First, regarding the difference between the task conditions, $\delta[0 x y-H b]$ at the bilateral frontal pole regions and left inferior frontal region in controls was higher during the free-drawing compared with copying task. This result is consistent with Igimi's study. ${ }^{18}$ On the other hand, in patients, there was no difference in $\delta[0 x y-H b]$ between the free-drawing and copying tasks. Regarding the difference between groups, $\delta[0 x y-H b]$ in patients during both free-drawing and copying tasks was significantly lower at the bilateral inferior frontal regions and left inferior parietal region compared with controls. At the left middle frontal region, right inferior parietal region, and left superior temporal region, $\delta[\mathrm{oxy}-\mathrm{Hb}]$ in patients was lower than that in the controls only during the free-drawing task.

The middle frontal region comprises the dorsolateral prefrontal cortex (DLPFC; BA 46/9), which is an important component of WM and the central executive function. ${ }^{24}$ This function is the mainstay of WM, which involves information processing while controlling other components: the phonological loop, visuospatial sketchpad, and episodic buffer, and it is also involved in focusing, suppression, and shifting of attention. ${ }^{25}$ In previous studies using WM tasks, lateralization of activation was demonstrated during a semantic fluency task in healthy subjects. ${ }^{26}$ Moreover, a decrease of lateralization was observed in patients with SZ during visuospatial WM tasks such as the TMT. ${ }^{15,27}$ Furthermore, activation at the middle frontal area reflecting the WM system has been reported in patients with SZ while concentrating (in a card game). ${ }^{23}$ Since the TMT and concentration require constant behavioral modification according to the purpose, the importance of not only WM but also the executive function is considered to be marked. Therefore, comparison between the controls and patients in the present study suggested that patients found it more difficult to activate the left middle frontal region related to the executive function during the free-drawing task than controls.

In present study, activation at the left inferior frontal region of patients was weaker than that of the controls during both the free-drawing and copying tasks. An fMRI study using the visual WM task also revealed that patients showed decreased activation of the ventrolateral prefrontal cortex (VLPFC) at the inferior frontal area at an early stage of maintenance after coding visual information. ${ }^{28}$ Baddeley stated that the episodic buffer of WM interacts with long-term memory. ${ }^{29}$ In addition, more detailed studies revealed that the inferior frontal area plays an important role in mnemonic retrieval and, notably, demonstrated that the area functions in top-down mnemonic retrieval. ${ }^{30,31}$ In the present study, based on the results of differences between task conditions in controls, it was considered that the free-drawing task to imagine a tree required more activation in the left inferior frontal region than the copying task. In addition, the activation at the inferior frontal region in patients was lower than that in controls during both the free-drawing and copying tasks. Therefore, it can be stated that the activation at the left inferior frontal region in patients shows a greater decrease during the free-drawing task than in controls. Thus, the abnormality of top-down retrieval to long-term memory associated with this region is important when considering the brain dysfunction of patients with SZ during the tree-drawing test. By the way, the frontal pole region has been reported as a region coordinating DLPFC and VLPFC. ${ }^{32,33}$ In the present study, the bilateral frontal pole activities during the free-drawing task in controls were greater than that during the copying task. Therefore, it was considered that the free-drawing task requires more coordination of the frontal pole.

The activation of the left and right inferior parietal regions during the free-drawing task in patients was also weaker than that in the controls. At the parietal cortex, previous studies revealed that the intraparietal sulcus (IPS) and inferior parietal lobule together with the superior parietal lobule are regions controlling spatial attention. ${ }^{34-37}$ Furthermore, concerning WM, a positron emission tomography (PET) study showed that the left inferior parietal region is related to phonological storage. ${ }^{38}$ For visuospatial WM, it has been demonstrated that activations at the bilateral superior parietal regions are related. ${ }^{39}$ Moreover, it was reported that the inferior parietal region is responsible for the visuospatial sketch pad and that the frontoparietal network is important for visuospatial WM. ${ }^{40}$ 
On the other hand, in patients with SZ, an fMRI study using the Sternberg task, which is a WM task to visually perceive letters, reported their decreased activation at the inferior parietal region. ${ }^{41}$ Therefore, it is suggested that the free-drawing task reveals the dysfunction of visuospatial attention or WM in patients with SZ.

$\delta[\mathrm{oxy}-\mathrm{Hb}]$ at the left superior temporal region during the free-drawing task in patients was also lower than that in controls. As shown in a previous study, the superior temporal region is considered to be involved in the retrieval of visual meaning, ${ }^{11}$ and the free-drawing task was considered to require retrieval of the meaning of trees. Also, activation at the prefrontal and temporal regions and verbal semantic retrieval are related. ${ }^{42}$ Considering that its dysfunction in patients with SZ has been reported, ${ }^{43}$ patients in the present study might have had problems with processing the meaning of trees during the free-drawing task.

Thus, we identified an association between the treedrawing task using free drawing and activation in the prefrontal or parietal region and temporal region. Some previous studies obtained results indicating that the frontoparietal network is important in WM and visuospatial attention. ${ }^{44} 46$ Furthermore, as indicated by studies in patients with unilateral spatial neglect, the dorsal attention network, in which the frontal eye field and IPS are connected, is involved in active attention, and the ventral attention network, in which the middle frontal area and temporoparietal junction are connected, is involved in passive attention. ${ }^{47-49}$ Recently, in patients with SZ, the results of a study showing functional abnormality at the frontoparietal network were reported. ${ }^{50-52}$ In addition, a study identified the relationship between a deficit in the maintenance of goals as an executive function and low-level activation at the frontoparietal network in patients with SZ. ${ }^{53}$ Although the examination of such functional connectivity requires an fMRI study, the decrease of $\delta[\mathrm{oxy}-\mathrm{Hb}]$ at the middle frontal, inferior frontal, inferior parietal, and superior temporal regions in patients observed in the present study suggests the dysfunction of the frontoparietal or frontaltemporal network.

Regarding the relationship between $\delta[\mathrm{oxy}-\mathrm{Hb}]$ and symptoms, activation at the left inferior frontal region during the free-drawing task was significantly positively correlated with both the positive and negative symptom scores in patients. In the copying task, activation at the left inferior frontal region was significantly negatively correlated with the positive symptom score and activation at the right middle frontal region was significantly negatively correlated with the negative symptom score. A PET study of untreated patients with SZ reported the presence of hypermetabolism at the prefrontal cortex when positive symptoms predominate. ${ }^{54}$ In addition, a meta-analysis of functional neuroimaging studies of the executive function in patients with $\mathrm{SZ}$ noted that activation at VLPFC was relatively increased, suggesting that it is a compensatory response related to decreased activation in the anterior cingulate gyrus, temporal cortex, and parietal cortex..$^{55}$ An NIRS study using word generation tasks also identified a positive correlation between the brain activation at the prefrontal region and positive symptom score, suggesting that it is a mechanism to compensate for inefficiency of the brain function. ${ }^{56}$ Therefore, from the above results, although there was no difference in $\delta[\mathrm{oxy}-\mathrm{Hb}]$ between the free-drawing and copying tasks in patients, during the freedrawing task, patients with severe positive symptoms may be in a state whereby other brain regions must be compensated for by activating the VLPFC.

As above, our NIRS study revealed that changes in [oxy-Hb] at the left middle frontal region, bilateral inferior frontal regions, bilateral inferior parietal regions, and left superior temporal region are low while drawing an imagined tree in patients with SZ. In particular, it should be noted that the left inferior frontal region, which should be more activated by the free-drawing task than the copying task in controls, was less active in patients. This suggests that mnemonic retrieval in patients with SZ may not properly function during the tree-drawing test. For that reason, patients with SZ may have a strong tendency to draw characteristic trees in the tree-drawing test, coupled with the abnormity of attention, executive function, and WM. Therefore, as we hypothesized initially, when interpreting the tree-drawing test of patients with SZ, it is necessary to consider not only the psychological and psychopathological aspects but also the influence of the cognitive function. Furthermore, the application of these findings may contribute to the development of the tree-drawing test as an objective assessment method in patients with SZ. Morita et al reported that malignant indices such as a small trunk-to-crown ratio and opening of the trunk are likely to appear in the tree-drawing test of patients with $\mathrm{SZ}$ and that this test is useful for evaluating time-dependent changes in symptoms. ${ }^{57}$ In future, by examining the association between changes in [oxy-Hb] during the tree-drawing test and such indices related to drawing contents or the results of other neuropsychological tests, a more objective method to assess the tree-drawing test may be identified. In addition, by observing time-dependent changes of these results, prediction of the symptom recovery and drug reactivity in patients with SZ based on the results of the tree-drawing test may become possible. In such a case, it will be necessary to consider the stages and severity of SZ. 
The present study had some limitations. First, the number of subjects was small, with 28 subjects in each group. Second, since there was a slight but significant difference between the IQ of healthy controls and patients with SZ, a difference in intellectual ability might have influenced the results. Therefore, further studies need to match the IQ of both the groups. Third, all patients had taken atypical antipsychotics, and so there is some possibility of an association with WM deficit. Meta-analysis studies investigating the influence of atypical antipsychotics on cognitive functions revealed that drugs reduce some cognitive functions and that the effect of improving the cognitive function is not uniform. ${ }^{58,59}$ Thus, future studies should consider drug-naive patients. Fourth, the tree-drawing task in the present study had methodological restrictions, such as setting a time limit, and so did not fully reflect the standard tree-drawing test. Therefore, in future studies, it would be preferable to adopt an actual test situation.

\section{Conclusion}

Our study showed that changes in [oxy-Hb] at the left middle frontal region, bilateral inferior frontal regions, bilateral inferior parietal regions, and left superior temporal region when drawing a tree imagined freely in the patients with SZ are smaller than that in healthy controls. These results suggested that brain dysfunction in patients with $\mathrm{SZ}$ is involved in their tree-drawing. In addition, it might contribute to more objective assessment method of the tree-drawing test.

\section{Acknowledgment}

The authors thank all subjects in the present study.

\section{Author contributions}

Nakano, Morita, and Igimi designed the study concept. Nakano drafted the manuscript. Nakano, Morita, Igimi, and Ishii were involved in the recruitment of subjects and data acquisition. Morita and Shoji diagnosed the patients. The statistical analysis was performed by Nakano and Morita, and the results were interpreted by all the authors. The study was supervised by Uchimura. Nakano and Shoji wrote the final version of the manuscript. All the authors contributed to the critical revision and approved the final manuscript.

\section{Disclosure}

The authors report no conflicts of interest in this work.

\section{References}

1. Bowie CR, Harvey PD. Cognitive deficits and functional outcome in schizophrenia. Neuropsychiatr Dis Treat. 2006;2(4):531-536.
2. Heilbronner U, Samara M, Leucht S, Falkai P, Schulze TG. The longitudinal course of schizophrenia across the lifespan: clinical, cognitive, and neurobiological aspects. Harv Rev Psychiatry. 2016;24(2):118-128.

3. Koch C. The Tree Test: the tree-drawing test as an aid in psychodiagnosis. Bern: Hans Huber; 1952.

4. Kaneda A, Yasui-Furukori N, Saito M, et al. Characteristics of the tree-drawing test in chronic schizophrenia. Psychiatry Clin Neurosci. 2010;64(2):141-148.

5. Stanzani MM, Matacena C, Sambati L, et al. The tree-drawing test (Koch's Baum test): a useful aid to diagnose cognitive impairment. Behav Neurol. 2015;2015:534681.

6. Panesi S, Morra S. Drawing a dog: the role of working memory and executive function. $J$ Exp Child Psychol. 2016;152:1-11.

7. Tulving E, Kapur S, Craik F, Moscovitch M, Houle S. Hemispheric encoding/retrieval asymmetry in episodic memory: positron emission tomography findings. Proc Natl Acad Sci US A. 1994;91(6):2016-2020.

8. Trojano L, Grossi D, Linden DE, et al. Matching two imagined clocks: the functional anatomy of spatial analysis in the absence of visual stimulation. Cereb Cortex. 2000;10(5):473-481.

9. Formisano E, Linden DE, Di Salle F, et al. Tracking the mind's image in the brain I: time-resolved fMRI during visuospatial mental imagery. Neuron. 2002;35(1):185-194.

10. Ino T, Asada T, Ito J, Kimura T, Fukuyama H. Parieto-frontal networks for clock drawing revealed with fMRI. Neurosci Res. 2003;45(1):71-77.

11. Harrington GS, Farias D, Davis CH. The neural basis for simulated drawing and the semantic implications. Cortex. 2009;45(3):386-393.

12. Ehlis AC, Herrmann M, Plichta M, Fallgatter A. Cortical activation during two verbal fluency tasks in schizophrenic patients and healthy controls as assessed by multi-channel near-infrared spectroscopy. Psychiatry Res. 2007;156(1):1-13.

13. Sato H, Dresler T, Haeussinger F, Fallgatter A, Ehlis A. Replication of the correlation between natural mood states and working memoryrelated prefrontal activity measured by near-infrared spectroscopy in a German sample. Front Hum Neurosci. 2014;8:37.

14. Otsuka Y. Face recognition in infants: a review of behavioral and nearinfrared spectroscopic studies. Jpn Psychol Res. 2013;56(1):76-90.

15. Fujiki R, Morita K, Sato M, et al. Reduced prefrontal cortex activation using the Trail Making Test in schizophrenia. Neuropsychiatr Dis Treat. 2013;9:675-685.

16. Pu S, Nakagome K, Itakura M, et al. Self-reported social functioning and prefrontal hemodynamic responses during a cognitive task in schizophrenia. Psychiatry Res. 2015;234(1):121-129.

17. Shoyama M, Nishioka T, Okumura M, et al. Brain activity during the clock-drawing test: multichannel near-infrared spectroscopy study. Appl Neuropsychol. 2011;18(4):243-251.

18. Igimi H. Changes in oxy-hemoglobin during tree drawing in healthy subjects evaluated by multichannel NIRS. Kyushu Neuropsychiatr. 2010;56:75-82. Japanese.

19. American Psychiatric Association. Diagnostic and Statistical Manual of Mental Disorders, 4th Edition, Text Revision (DSM-IV-TR). Washington, DC: American Psychiatric Association; 2000.

20. Matsuoka K, Kin Y. Japanese Adult Reading Test (JART). Tokyo: Shinko Igaku Syuppansya; 2007.

21. Kay SR. Positive and Negative Syndromes in Schizophrenia. Abingdonon-Thames: Routledge Mental Health; 1991.

22. Oldfield RC. The assessment and analysis of handedness: the Edinburgh inventory. Neuropsychologia. 1971;9(1):97-113.

23. Fujiki R, Morita K, Inoue M, et al. Characteristics of cortical activation in schizophrenia during the card game "concentration". Kurume Med J. 2012;59(34):53-60.

24. Baddeley AD, Hitch GJ. Working memory. In: Bower GH, editor. The psychology of learning and motivation: Advances in research and theory. New York: Academic Press. 1974:47-89.

25. Smith E, Jonides J. Working memory: a view from neuroimaging. Cogn Psychol. 1997;33(1):5-42.

26. Tupak SV, Badewien M, Dresler T, et al. Differential prefrontal and frontotemporal oxygenation patterns during phonemic and semantic verbal fluency. Neuropsychologia. 2012;50(7):1565-1569. 
27. Lee J, Folley BS, Gore J, Park S. Origins of spatial working memory deficits in schizophrenia: an event-related FMRI and near-infrared spectroscopy study. PLoS One. 2008;3(3):e1760.

28. Grot S, Légaré VP, Lipp O, Soulières I, Dolcos F, Luck D. Abnormal prefrontal and parietal activity linked to deficient active binding in working memory in schizophrenia. Schizophr Res. 2017;188:68-74.

29. Baddeley A. The episodic buffer: a new component of working memory? Trends Cogn Sci. 2000;4(11):417-423.

30. Badre D, Poldrack RA, Paré-Blagoev EJ, Insler RZ, Wagner AD. Dissociable controlled retrieval and generalized selection mechanisms in ventrolateral prefrontal cortex. Neuron. 2005;47(6):907-918.

31. Badre D, Wagner AD. Left ventrolateral prefrontal cortex and the cognitive control of memory. Neuropsychologia. 2007;45(13):2883-2901.

32. D'Esposito M, Detre JA, Alsop DC, Shin RK, Atlas S, Grossman M. The neural basis of the central executive system of working memory. Nature. 1995;378(6554):279-281.

33. Bisconti S, Di Sante G, Ferrari M, Quaresima V. Functional nearinfrared spectroscopy reveals heterogeneous patterns of language lateralization over frontopolar cortex. Neurosci Res. 2012;73(4):328-332.

34. Hopfinger JB, Buonocore MH, Mangun GR. The neural mechanisms of top-down attentional control. Nat Neurosci. 2000;3(3):284-291.

35. Bisley JW, Goldberg ME. Neuronal activity in the lateral intraparietal area and spatial attention. Science. 2003;299(5603):81-86.

36. Chiu YC, Yantis S. A domain-independent source of cognitive control for task sets: shifting spatial attention and switching categorization rules. J Neurosci. 2009;29(12):3930-3938.

37. Wang J, Yang Y, Fan L, et al. Convergent functional architecture of the superior parietal lobule unraveled with multimodal neuroimaging approaches. Hum Brain Mapp. 2015;36(1):238-257.

38. Paulesu E, Frith CD, Frackowiak RS. The neural correlates of the verbal component of working memory. Nature. 1993;362(6418):342-345.

39. Fiez JA, Raife EA, Balota DA, Schwarz JP, Raichle ME, Petersen SE. A positron emission tomography study of the short-term maintenance of verbal information. J Neurosci. 1996;16(2):808-822.

40. Kwon H, Reiss AL, Menon V. Neural basis of protracted developmental changes in visuo-spatial working memory. Proc Natl Acad Sci U S A. 2002;99(20):13336-13341.

41. Wible CG, Lee K, Molina I, et al. fMRI activity correlated with auditory hallucinations during performance of a working memory task: data from the FBIRN consortium study. Schizophr Bull. 2009;35(1):47-57.

42. Cabeza R, Nyberg L. Imaging cognition II: an empirical review of 275 PET and fMRI studies. J Cogn Neurosci. 2000;12(1):1-47.

43. Jennings JM, McIntosh AR, Kapur S, Zipursky RB, Houle S. Functional network differences in schizophrenia: a rCBF study of semantic processing. Neuroreport. 1998;9(8):1697-1700.

44. Xu GQ, Lan Y, Huang DF, et al. Visuospatial attention deficit in patients with local brain lesions. Brain Res. 2010;1322:153-159.
45. Buckner RL, Krienen FM, Yeo BT. Opportunities and limitations of intrinsic functional connectivity MRI. Nat Neurosci. 2013;16(7): $832-837$.

46. Nummenmaa L, Oksama L, Glerean E, Hyönä J. Cortical circuit for binding object identity and location during multiple-object tracking. Cereb Cortex. 2017;27(1):162-172.

47. Corbetta M, Shulman GL. Control of goal-directed and stimulus-driven attention in the brain. Nat Rev Neurosci. 2002;3(3):201-213.

48. He BJ, Snyder AZ, Vincent JL, et al. Breakdown of functional connectivity in frontoparietal networks underlies behavioral deficits in spatial neglect. Neuron. 2007;53(6):905-918.

49. Vossel S, Geng JJ, Fink GR. Dorsal and ventral attention systems: distinct neural circuitsbut collaborative roles. Neuroscientist. 2014; 20(2):150-159.

50. Tu PC, Lee YC, Chen YS, Li CT, Su TP. Schizophrenia and the brain's control network: aberrant within- and between-network connectivity of the frontoparietal network in schizophrenia. Schizophr Res. 2013; 147(2-3):339-347.

51. Phillips RC, Salo T, Carter CS. Distinct neural correlates for attention lapses in patients with schizophrenia and healthy participants. Front Hum Neurosci. 2015;9:502.

52. Nielsen JD, Madsen KH, Wang Z, Liu Z, Friston KJ, Zhou Y. Working memory modulation of frontoparietal network connectivity in firstepisode schizophrenia. Cerebral Cortex. 2017;27(7):3832-3841.

53. Poppe AB, Barch DM, Carter CS, et al. Reduced frontoparietal activity in schizophrenia is linked to a specific deficit in goal maintenance: a multisite functional imaging study. Schizophr Bull. 2016;42(5): 1149-1157.

54. Shinto AS, Kamaleshwaran KK, Srinivasan D, et al. "Hyperfrontality" as seen on FDG PET in unmedicated schizophrenia patients with positive symptoms. Clin Nucl Med. 2014;39(8):694-697.

55. Minzenberg MJ, Laird AR, Thelen S, Carter CS, Glahn DC. Metaanalysis of 41 functional neuroimaging studies of executive function in schizophrenia. Arch Gen Psychiatry. 2009;66(8):811-822.

56. Fujiki R, Morita K, Sato M, et al. Single event-related changes in cerebral oxygenated hemoglobin using word game in schizophrenia. Neuropsychiatr Dis Treat. 2014;10:2353-2360.

57. Morita K, Nakamura H, Hara K. Evaluation and classification of psychiatric disorder: analysis of time-dependent changing in the Baum test. Seishinka Chiryougaku. 1998;13(10):11249-11256. Japanese.

58. Nielsen RE, Levander S, Kjaersdam Telle'us G, et al. Second-generation antipsychotic effect on cognition in patients with schizophrenia - a meta-analysis of randomized clinical trials. Acta Psychiatr Scand. 2015;131(3):185-196.

59. Steen NE, Aas M, Simonsen C, et al. Serum levels of second-generation antipsychotics are associated with cognitive function in psychotic disorders. World J Biol Psychiatry. 2017;18(6):471-482.
Neuropsychiatric Disease and Treatment

\section{Publish your work in this journal}

Neuropsychiatric Disease and Treatment is an international, peerreviewed journal of clinical therapeutics and pharmacology focusing on concise rapid reporting of clinical or pre-clinical studies on a range of neuropsychiatric and neurological disorders. This journal is indexed on PubMed Central, the 'PsycINFO' database and CAS,
Dovepress

and is the official journal of The International Neuropsychiatric Association (INA). The manuscript management system is completely online and includes a very quick and fair peer-review system, which is all easy to use. Visit http://www.dovepress.com/testimonials.php to read real quotes from published authors. 